\title{
Importancia de la psicología de la salud en la atención primaria, promoción y prevención de la salud
}

\section{Importance of health psychology in primary care, health promotion and prevention. Rodrigo Rodríguez-Terán ${ }^{a}$}

\begin{abstract}
:
Health psychology has been developing for almost five decades since its consolidation as a scientific discipline, which is why it has faced different challenges in order to introduce itself into the field of health sciences, specifically as a support in the areas of medicine. This paper starts from an ambiguous and unreal concept of what health is, the process of consolidation of health psychology as a science, the contributions in terms of primary care, health promotion and prevention, to the challenges that this discipline faces today. Finally, it is concluded that health psychology will be the tool that will mark a before and after in the interventions that are carried out in the areas that encompass the health-disease process.
\end{abstract}

\section{Keywords:}

Psychology, Health, Primary health care, Health Promotion, Behavioral Medicine

\section{Resumen:}

La psicología de la salud se ha venido desarrollando desde hace ya casi cinco décadas desde su consolidación como disciplina científica, por lo cual, se ha enfrentado a distintos retos para lograr introducirse en el campo de las ciencias de la salud, específicamente como apoyo en las áreas de la medicina. El presente documento parte desde un concepto ambiguo e irreal de lo que es la salud, el proceso de consolidación de la psicología de la salud como ciencia, los aportes en materia de atención primaria, promoción y prevención de la salud, hasta los retos que se le presentan en la actualidad para esta disciplina. Finalmente, se concluye que la psicología de la salud será la herramienta que marcará un antes y un después en las intervenciones que se realicen en las áreas que engloban el proceso de salud-enfermedad.

\section{Palabras Clave:}

Psicología, Salud, Atención primaria de salud, Promoción de la salud, Medicina de la Conducta

\section{Introducción}

Históricamente, la necesidad que tiene el ser humano de darle una interpretación y significado a los fenómenos que ocurren a su alrededor, en este caso, de la salud y la enfermedad, ha desembocado en dos propuestas: las que se enfocan en el estudio del por qué las personas se enferman y aquellas que enfatizan en la promoción de la salud y la prevención de las afecciones/enfermedades [1].
Estas propuestas se remontan a la época griega, en donde se desarrollan dos escuelas enfocadas en demostrar el origen de la enfermedad: la escuela platónica y la escuela hipocrática. La primera, hace referencia a que "la enfermedad es un castigo divino de los dioses que se curaba no con medicamentos, sino con ritos sagrados", mientras que la segunda, propone que las enfermedades

\footnotetext{
Autor de Correspondencia, Universidad Privada del Estado de México, https://orcid.org/0000-0002-1821-2066, Email: roll.royce9910@gmail.com
} 
tienen un origen natural, libre de toda concepción divina. [1]

Fue durante mucho tiempo que el concepto de salud estuvo sujeto a interpretaciones variadas (religión y filosofía principalmente), además de considerarse a la salud únicamente como la ausencia de enfermedades. Es hasta 1946 que la Organización Mundial de la Salud (OMS) declara que la salud "es un estado de completo bienestar físico, mental y social, y no solamente la ausencia de afecciones o enfermedades" [2].

\section{Hacia un nuevo concepto de salud}

El concepto de salud que ha propuesto la OMS es irrealista, debido a que es prácticamente imposible alcanzar un bienestar completo y estable, por lo que resulta necesario establecer una definición más precisa sobre lo que es la salud como tal, al respecto Blum plantea un modelo de ambiente de salud conformado por cuatro componentes (biología humana, medio ambiente, estilo de vida y organización de la atención de la salud), los cuales se relacionan y modifican entre sí dentro del círculo poblacional [3].

Con base en este modelo, se ha logrado concebir a la salud como un "proceso de variaciones ininterrumpidas que acompañan el fenómeno vital de la persona, las cuales son producidas 0 influidas por factores hereditarios, de comportamiento y ambientales, así como por acciones provenientes de los servicios de salud" [4] en lugar de un "estado de completo bienestar".

A raíz de la nueva conceptualización sobre lo que es la salud en general, la psicología comienza a adentrarse más en el análisis del proceso de salud-enfermedad, dando como resultado la consolidación del campo de la psicología de la salud alrededor de la década de los setenta [5-6].

\section{Génesis: Un nuevo campo disciplinar}

En un principio, la psicología de la salud se empezaba a definir como "una parte de la psicología clínica, que surge a partir del interés en el ámbito médico, como si el trabajo en el campo de la salud fuera reductible a un espacio tan limitado como la mera asistencia y cura de enfermedades" [7]. Sin embargo, no es posible concebir a la psicología de la salud como una parte de la psicología clínica, ya que no se enfoca solamente en los trastornos mentales, sino que va más allá de éstos.

Al respecto, Piña y Rivera proponen que la psicología de la salud debe entender como un campo de actuación profesional, donde las psicólogas y los psicólogos deben poseer un conjunto integral de conocimientos y competencias que les permitan cumplir con la investigación de qué y cómo es que las variables psicológicas facilitan o dificultan las conductas de riesgo y prevención de contraer una enfermedad, así como la promoción de la salud [6].

\section{Desarrollo}

\section{Psicología de la salud: Matarazzo.}

Joseph Dominic Matarazzo ha sido el pionero en el desarrollo de la medicina conductual y su relación con la salud conductual, debido a esto, sus aportes para la psicología de la salud resultan imprescindibles para las y los profesionales de la psicología.

El principal aporte que realiza Matarazzo a la psicología de la salud es determinarla como "la suma de contribuciones específicas educativas, científicas y profesionales de la psicología para la prevención y tratamiento de las enfermedades, promoción y mantenimiento de la salud, identificación de los correlatos etiológicos y diagnósticos de la salud, la enfermedad y la disfunción relacionada, así como el análisis y mejora del sistema de atención de la salud y la formación de políticas de salud" [8].

\section{El objeto de interés}

A partir de la concepción que hace Matarazzo, la psicología de la salud se deberá interesar más en dos aspectos, "el primero será sobre todas aquellas conductas asociadas a la adquisición, desarrollo y mantenimiento de enfermedades o de un estado de salud óptimo, y el segundo referente a los factores psicológicos derivados del diagnóstico y tratamiento de una enfermedad física, además de las implicaciones que estos conlleven a nivel emocional en la persona" [9].

Además de esto, es importante mencionar que la diferencia entre los demás campos de la psicología y el propio de la psicología de la salud, radica en que cualquier actividad de la psicología, que tenga relación con los procesos de salud-enfermedad, principalmente en el aspecto físico, pertenece al campo de psicología de la salud [7], un ejemplo de esto sería la elaboración de políticas sanitarias y lo referente a los procesos de atención primaria, promoción y prevención de la salud. 


\section{Atención primaria de la salud}

Es necesario que la psicología de la salud se adentre en el terreno de la Atención Primaria de la Salud (APS), esto debido a que la APS refiere a todos aquellos mecanismos que son factores determinantes de la salud (políticos, culturales, financieros, sanitarios, entre otros) que son potencialmente efectivos para que la población en general pueda acceder a los servicios de asistencia básicos con bajos costos y que sean de calidad [10-11].

Esta importancia radica en que la psicología de la salud evidencia beneficios inmensos dentro de la APS más allá de mantenerse en el campo de acción de la psicología clínica, es decir, en terapias psicológicas. Dentro de los beneficios que aportaría la psicología de la salud en la APS encontramos mejoría en el control y seguimiento de enfermedades crónicas con componentes psicológicos importantes, su accesibilidad es mayor al encontrarse en centros de salud por lo que el costo para la población sería menor, además de contar con un enfoque biopsicosocial que permite una mayor precisión de los factores desencadenantes de la enfermedad y las conductas saludables para el mantenimiento de un equilibrio óptimo del organismo.

Estos beneficios se han demostrado en diversos proyectos enfocados en la atención de adultos con trastornos mentales, uno de estos proyectos ha sido implementado en Reino Unido [12] que, aunque dirigido al campo de la psicología clínica, nos permite vislumbrar el qué pasaría si se comenzara a implementar, de manera prioritaria, la psicología de la salud en la APS.

\section{Promoción de la salud}

Retomando lo expuesto en la Carta de Ottawa [13], la Promoción de la Salud (PS) deberá consistir en poner al alcance de los pueblos los medios necesarios para incrementar su salud y poder llevar un control mayor sobre la misma, esto con la finalidad de que la persona esté capacitada para obtener un estado apropiado de bienestar biopsicosocial, mediante el reconocimiento y consumación de sus aspiraciones, la satisfacción de sus necesidades y su capacidad de resiliencia

Es menester que la psicología de la salud se vea involucrada en los procesos de promoción de conductas y hábitos saludables para la obtención de un estado equilibrado del organismo en su totalidad, esto conlleva un esfuerzo importante en el desarrollo de elementos que sirvan para la promoción de la salud en las personas.
Una herramienta factible de implementar para generar la promoción de conductas y hábitos saludables sería la educación para la salud, esto debido a que permite visualizar la promoción de la salud como una dinámica de enseñanza-aprendizaje, la cual otorga la posibilidad de utilizar los recursos disponibles de una población para generar estilos de vida más óptimos y con mucho mayor nivel de bienestar a bajo costo y de calidad para las personas [14].

La funcionalidad que tiene la psicología de la salud gracias a la formación profesional que ofrece, además de su efectividad para promover conductas que favorecen el equilibrio biopsicosocial de las personas, apertura múltiples posibilidades de implementación para apoyar a los esfuerzos conjuntos de todas las instituciones nacionales e internacionales para la promoción de la salud en la población, de acuerdo con lo estipulado en la Declaración de Alma-Ata y la Carta de Ottawa $[11,13]$.

\section{Prevención de la salud}

A diferencia de la promoción de la salud, la prevención de la salud o también llamada prevención de enfermedades, hace referencia a las intervenciones que tienen por objetivo reducir o eliminar los comportamientos que se pueden calificar como factor de riesgo para el desarrollo de algún tipo de enfermedad [15].

No obstante, no se limitará únicamente a los factores de riesgo, sino que además se analizará a las poblaciones de riesgo, ya que es imprescindible comprender la dinámica social que presentan las personas que adquieren una enfermedad, ya sea infecto-contagiosas 0 crónicodegenerativas. Esto posibilita que las y los profesionales de la psicología de la salud puedan generar pautas de acción más precisas para reducir los índices de infección y/o mortalidad, además de proporcionar evidencia para ser utilizada en medidas de prevención y promoción futura.

\section{Retos de la psicología de la salud}

A pesar de los múltiples beneficios y oportunidades que proporciona la psicología de la salud en materia de APS, la PS y la prevención de la salud, los retos que debe superar para ser aceptada como una disciplina científica, capaz de formar parte de un campo que hasta ahora ha sido exclusivo de la medicina, son variados, pero que principalmente radican en la transformación de paradigmas, políticas y servicios de salud para volverlos un campo multi e interdisciplinario, pues esto, no depende de las y los profesionales de la psicología de la salud [16]. 
Otro reto visible para la psicología de la salud es que la población y las políticas públicas que rigen el sistema de salud, no han podido identificar los aportes que tiene esta disciplina científica para prevenir enfermedades, promocionar la salud y aumentar el nivel de calidad de vida tanto de manera individual como poblacional. La raíz de este reto quizás radique en la falta de claridad de la o el profesional en relación al campo de acción en donde se desarrollará 0 las herramientas que utilizará, que finalmente son propias de la psicología como tal y su fundamento se encuentra en la evidencia que otorga a partir de las múltiples investigaciones que se han hecho con base en el método científico [17].

\section{Conclusión}

Cabe destacar que la psicología de la salud, a casi cinco décadas de su consolidación como disciplina científica, ha realizado múltiples aportaciones en diversos países a nivel mundial en materia de salud pública. No obstante, aún se enfrenta ante la incertidumbre que puede causar el contexto sociopolítico cambiante del país donde se implemente.

Ante tal situación, la psicología de la salud deberá aumentar sus esfuerzos por seguir coadyuvando a las diferentes áreas de la salud (como lo es la medicina, la nutrición, la epidemiología, entre otras), ya que sin el conocimiento que aporta de manera teórica y metodológica proveniente de la psicología, las demás disciplinas perderían sentido y dirección al momento de establecer una óptima APS, PS y prevención de las enfermedades [17].

Es por esto que, más que desalentar a las y los estudiantes que buscan especializarse en la psicología de la salud con los retos y responsabilidades que el área exige, se incentiva a que sean factores de cambio en la concepción de la APS, la implementación de una PS efectiva y los medios para prevenir la adquisición de enfermedades.

Finalmente, la psicología de la salud juega un papel fundamental en el futuro de la población ya que, como se ha venido mencionando a lo largo del escrito, sus aportaciones e implicaciones en el proceso de saludenfermedad son vitales para generar una dinámica de bienestar óptimo en las personas.

En conclusión, se puede considerar a esta noble disciplina como la herramienta necesaria para la modificación de los patrones conductuales de riesgo para la salud, la eliminación del estigma de la atención en materia de salud mental y su relación con las enfermedades físicas, el cambio en el paradigma médico sobre la exclusividad del área y la conceptualización de la psicología como parte de las ciencias de la salud, además de marcar un antes y un después en la dinámica de la APS, PS y prevención de la enfermedad a partir de la práctica basada en evidencia.

\section{Referencias}

[1] Díaz, V. La psicología de la salud: antecedentes, definición y perspectivas. rev. psicol. univ. antioquia. 2010; 2(3): 57-71. Disponible en: http://pepsic.bvsalud.org/pdf/rpsua/v2n1/v2n1a5.pdf

[2] Organización Mundial de la Salud [Página principal en internet]. Ginebra; [citado 08 abril 2021] OMS. Constitución [aprox. 3 pantallas]. Disponible en: https://www.who.int/es/about/who-we-are/constitution

[3] Álvarez, A., García, A., Bonet, M. Pautas conceptuales y metodológicas para explicar los determinantes de los niveles de salud en Cuba. Rev Cubana Salud Pública. 2007; 33(2): 1-16. Disponible en: http://scielo.sld.cu/pdf/rcsp/v33n2/spu13207.pdf

[4] Flórez-Alarcón, L. Psicología social de la salud. Promoción y prevención. México: Manual Moderno; 2007.

[5] Morales, F. Psicología de la salud. Realizaciones e interrogantes tras cuatro décadas de desarrollo. PSIENCIA. Revista Latinoamericana de Ciencia Psicológica. 2012; 4(2): 98-107. Disponible en: https://www.redalyc.org/articulo.oa?id=333127382005

[6] Piña, J., Rivera, B. Psicología de la salud: algunas reflexiones críticas sobre su qué y su para qué. Univ. Psychol. 2006; 5(3): 669-679. Disponible en: http://www.scielo.org.co/pdf/rups/v5n3/v5n3a19.pdf

[7] Contreras, F., Londoño, C., Vinaccia, S., Quiceno, J. Perspectivas de la psicología de la salud en Colombia. Investigación y Educación en Enfermería. 2006; 24(2): 120-129. Disponible en: https://www.redalyc.org/pdf/1052/105215402012.pdf

[8] Cerezo-Reséndiz, S., Hurtado-Rodríguez, C. Principales aportaciones a la medicina conductual de Joseph Dominic Matarazzo. Journal of Behavior, Health \& Social Issues. 2011-2012; 3(2): 49-59. Disponible en: https://www.redalyc.org/pdf/2822/282221802004.pdf

[9] Palacios, X., Pérez-Acosta, A. Evolución del concepto de psicología de la salud y su diferenciación de otros campos de acción profesional. Doc. Inv. Esc. Med. Cs. Salud. 2017; 24: 5-29. Disponible en: https://repository.urosario.edu.co/bitstream/handle/10336/12870/bi\%2024 $\%$ 20rehabilitación\%20web.pdf?sequence $=1$

[10] Organización Mundial de la Salud [Página principal en Internet]. Ginebra. OMS. Temas de salud: Atención primaria de salud [aprox. 3 pantallas]. Disponible https://www.who.int/topics/primary_health_care/es/

[11] Conferencia Internacional sobre Atención Primaria de Salud. Declaración de Alma-Ata. Almaty. 1978. Recuperado el 08 de abril de 2021, de https://www.paho.org/hq/dmdocuments/2012/Alma-Ata1978Declaracion.pdf

[12] Turpin, G., Richards, D., Hope, R., Duffy, R. Mejorando el acceso a las terapias psicológicas: una iniciativa nacional para asegurar la prestación de terapias de alta calidad basadas en la evidencia. Papeles del Psicólogo. 2008; Vol. 29(3): 271-280. Disponible en: http://www.papelesdelpsicologo.es/pdf/1609.pdf

[13] Ottawa Charter for Health Promotion. First International Conference on Health Promotion. Ottawa; 17-21 November 1986, obtenido de: https://www.paho.org/hq/dmdocuments/2013/Carta-de-ottawa-para-laapromocion-de-la-salud-1986-SP.pdf

[14] Díaz, Y., Pérez, J., Báez, F., Conde, M. Generalidades sobre promoción y educación para la salud. Revista Cubana de Medicina General Integral. 2012; 28(3): 299-308. Disponible http://scielo.sld.cu/pdf/mgi/v28n3/mgi09312.pdf 
Publicación semestral, Educación y Salud Boletín Científico Instituto de Ciencias de la Salud Universidad Autónoma del Estado de

Hidalgo, Vol. 10, No. 19 (2021) 49-53

[15] Amigo, I., Fernández, C., Pérez, M. Manual de psicología de la salud. 3a. ed. Madrid: Pirámide. 2009.

[16] Morales, F. Psicología de la salud. Realizaciones e interrogantes tras cuatro décadas de desarrollo. PSIENCIA. Revista Latinoamericana de Ciencia Psicológica. 2012; 4(2): 98-107. Disponible en: https://www.redalyc.org/pdf/3331/333127382005.pdf

[17] Pérez-Laborde, L., Moreyra-Jiménez, L. El quehacer del profesional de la psicología de la salud: Definiciones y objetivos. Revista Digital Internacional de Psicología y Ciencia Social. 2017; 3(2): 136-155. DOI: http://dx.doi.org/10.22402/j.rdipycs.unam.3.2.2017.81.136-155 